\title{
Family influences on physical activity and sedentary behaviours in Chinese junior high school students: a cross-sectional study
}

Xin Wang ${ }^{1}$, Qing-Min Liu' ${ }^{2}$ Yan-Jun Ren ${ }^{3}$, Jun Lv ${ }^{4^{*}}$ and Li-Ming Li ${ }^{5}$

\begin{abstract}
Background: Family influence plays an important role in a child's physical activity (PA). This study aimed to describe the level of moderate to vigorous intensity physical activity (MVPA) and sedentary behaviours among Chinese junior high school students and examine the associations between different types of family influence and MVPA or sedentary behaviours.

Methods: Participants of two independent cross-sectional surveys, conducted in 2009 and 2011, were students in Grade 7 and 9 from all junior high schools in Hangzhou, China. The daily duration and frequency of MVPA, amount of sedentary time and frequency of family support were self-reported. Multi-level mixed-effects logistic regression was used to examine the associations between different types or levels of family influence and MVPA or sedentary behaviours.

Results: A total of 7286 students were analysed finally. Overall, only $9.0 \%$ of the students participated in MVPA at least 60 minutes/day; 63.9\% spent no more than 2 hours/day in sedentary behaviours. Frequent verbal encouragement and watching were associated with less leisure-time sedentary behaviours. The multivariate-adjusted odds ratios (ORs) for verbal encouragement and watching were 1.29 (95\% Cl, 1.08 to 1.55) and 1.19 (95\% Cl, 0.97 to 1.45) for 5-7 days per week. The involvement of family in the children's activity in most days of the week was associated with both higher level of MVPA and less leisure-time sedentary behaviours. The respective ORs among students who reported familial support 5-7 days per week, were 1.50 (95\% Cl, 1.21 to 1.86) for engaging in seven days of MVPA per week, 1.67 (95\% Cl, 1.19 to 2.32) for at least 60 minutes of MVPA daily, and 1.48 ( $95 \%$ Cl, 1.19 to 1.84) for no more than 2 hours of leisure-time sedentary behaviours daily.
\end{abstract}

Conclusions: This study found that less than 10.0\% of urban Chinese adolescents engaged in MVPA at least 60 minutes/ day. Family involving themselves in the children's activity exerted the most significant influence on children's behaviours as compared with verbally encouraging and observing children's activity. Any type of familial support including verbally encouraging, watching, and involving had effects on reducing leisure-time sedentary behaviours.

Keywords: Moderate to vigorous intensity, Physical activity, Sedentary, Family influence, Adolescents

\footnotetext{
* Correspondence: Ivjun@bjmu.edu.cn

${ }^{4}$ Department of Epidemiology and Biostatistics, School of Public Health,

Peking University Health Science Centre, Beijing 100191, China

Full list of author information is available at the end of the article
} 


\section{Background}

Lack of physical activity (PA) and sedentary behaviours have been identified as risk factors for obesity, type 2 diabetes, cardiometabolic diseases, and depression [1-4]. Regular PA and reducing sitting time are associated with psychological benefits and better social development among adolescents. Also, physically active adolescents are more likely to avoid tobacco, alcohol, and drugs and therefore achieve better academic performance at school [5].An active lifestyle is not only necessary for physical and mental health in adolescence but also undoubtedly carried over into adulthood. Despite common knowledge on active lifestyle, a rapid decline in PA and an increase in sitting time have been observed in Chinese adolescents [6]. Over $50 \%$ of primary and secondary students in China spent less than one hour per day engaged in PA [7] and over 40\% students spent more than two hours a day in screen-based activity [6].

Several studies have correlated PA of adolescents with their family support such as parents encouraging their children to be more physical active, watching their children engaging in PA, and involving themselves in their children's activity [8-13]. These supports have been suggested to affect children's behaviours differently [14]. However, instead of identifying their effects separately, previous studies mostly treated them as a whole.

Therefore, we aimed to (1) describe the levels of moderate to vigorous intensity physical activity (MVPA) and sedentary behaviours in a sample of junior high school students in Hangzhou of China; (2) examine the associations between different types of family support and MVPA and sedentary behaviours among Chinese adolescents. The data of this article were part of the data collected in a multinational collaboration programme of the Community Interventions for Health $(\mathrm{CIH})$ [15]. The primary hypotheses in this analysis are that (1) family support is one of the most important motivational factors for children to move more and sit less; (2) children are motivated differently by different levels of support from verbally encouraging, watching, to involving.

\section{Methods}

\section{Subjects and sampling methods}

Participants were students in Grade 7 and 9 from all junior high schools in Xiacheng, Gongshu, and Xihu districts of Hangzhou City. Two independent crosssectional surveys were conducted in 2009 and 2011 in the same schools. Both of surveys applied same sample size requirement and sampling design. The sample size estimation was based on the primary aim of $\mathrm{CIH}$ programme, that was, intervention group exposed to interventions regarding tobacco use, food choices, and PA had a $6 \%$ greater change in the prevalence of each of the three risk factors [15]. The final sample size was arrived at using knowledge of prevalence of the three risk factors in cities of China. The largest sample size across all three risk factors was selected as the necessary sample size. In addition, $20 \%$ was added to allow for non-response. The sample size for each of survey was 4800 , with an equal size of 2400 subjects in grade 7 or 9. Stratified cluster sampling was used in both of the surveys. The list of classrooms was stratified by district, school, and grade. A simple random sample of classrooms were selected and all students in the selected classroom were eligible for the survey. Sampling fraction, that is, the ratio of sample size to population size, was applied to each strata to determine the number of classrooms and at least two classrooms in each strata were chosen. A total of 9271 students were investigated and the response rate was $94.8 \%$. The study was approved by the Institutional Review Board at Peking University Health Science Centre (IRB0000105208003). Informed consent ensuring privacy and confidentiality was obtained from both parent (or guardian) and student.

\section{Measures and variables}

Each student completed the $\mathrm{CIH}$ Youth Survey questionnaire addressing knowledge of, attitudes to, and behaviours in relation to unhealthy diet, physical inactivity, and tobacco use [15]. CIH Youth Survey was designed based on the Health Behaviour in School-aged Children (HBSC) [16]. While formal reliability and validity survey were not conducted in this population, this questionnaire has been shown to have good reliability and validity in other Chinese children [17]. Structured questionnaires were self-administered to the students. Physical examinations including height and weight were measured by trained field staff. The definitions of variables analysed in this article and their grouping methods are described as follows.

\section{Assessment of PA and sedentary behaviours}

The MVPA (including any activity that makes you sweat, get out of breath, and increases your heart rate, such as sports, school activities, playing with friends, and walking/ cycling to or from school) was assessed using the following two questions: (1) How many days per week on average do you participate in MVPA (at least 30 minutes) $(0-7$ days); and (2) How many minutes per day on average do you participate in MVPA in the aforementioned days $(30-39 \mathrm{mi}-$ nutes, $40-49$ minutes, $50-59$ minutes, or $\geq 60$ minutes). According to global recommendations on PA, children and youth aged 5-17 should accumulate at least $60 \mathrm{mi}-$ nutes of MVPA daily [18]. Therefore, the frequency ( $<7$ days/week or 7 days/week) and duration ( $<60 \mathrm{mi}-$ nutes/day or $\geq 60$ minutes/day) of MVPA were dichotomised. In addition, the total level of MVPA per week 
was dichotomized as follows: (1) meeting the recommendation: engaging in at least 60 minutes of MVPA daily; or (2) not meeting the recommendation: less than 7 days of MVPA per week or less than 60 minutes of MVPA per day. Sedentary behaviours were assessed using two questions as follows: (1) on school days, how much time per day on average do you spend in sedentary behaviours for leisure, including using a computer for pleasure, watching television/videos, playing electronic games, sitting and chatting, or other sitting activities (0 hours/day $-\geq 7$ hours/day); and (2) on weekends, how much time per day on average do you spend in sedentary behaviours for leisure( 0 hours/day $-\geq 7$ hours/ day). According to the global guidelines on youth sedentary behaviour, youth aged 12-17 should limit recreational screen time to no more than 2 hours per day [19]. Thus, the amount of sedentary time on school days and weekends was dichotomized as follows: (1) meeting the recommendation: no more than 2 hours of leisuretime sedentary behaviours per day; or (2) not meeting the recommendation: more than 2 hours of leisure-time sedentary behaviours per day.

\section{Assessment of family support}

Family support was assessed by the following three questions: (1) how many days per week on average do your families encourage you to engage in PA; (2) how many days per week on average do your families watch you engaging in PA; and (3) how many days per week on average do your families involve themselves in your activities, that is, having PA together with you. Response options included: (1) never or less than weekly; (2) 1-2 days/week; (3) 3-4 days/week; (4) 5-6 days/week; and (5) 7 days/week. The last two options were combined in the analyses.

\section{Assessment of other covariates}

Sex, grade (grade 7 or grade 9), mother's level of education (junior high school or below, senior high school, college or above, or don't know), health belief, and their perception of the neighbourhood environment, were reported by all students. BMI was calculated as weight in kilograms divided by the square of height in meters. Health belief was assessed by the following question: "To stay healthy, at least how much time per day should children like your age engage in PA" (15 minutes, $30 \mathrm{mi}-$ nutes, 60 minutes, 90 minutes, or do not know/not sure). We grouped health belief into two categories: (1) less than 60 minutes or do not know/not sure; or (2) at least 60 minutes. The perceptions of the neighbourhood environment were assessed by the following three questions: (1) it is unsafe to walk or jog because of the busy traffic and no walking trails around my neighbourhood; (2) parks or gyms are easily accessible around my neighbourhood; and (3) it was safe to walk or jog around my neighbourhood during the day. Response options for these three questions were as follows: (1) disagree strongly, (2) disagree slightly, (3) not sure, (4) agree slightly, or (5) agree strongly. We grouped the perceptions of the neighbourhood environments into three categories (agree, disagree, or not sure) in the analyses.

\section{Statistical analyses}

Chi-square tests were used to compare the counts of categorical responses between two or more independent groups. Nonparametric tests of K-independent samples were performed on the equality of the medians for the continuous variables without normal distribution. Statistical significance was defined as $\mathrm{P}<0.05$. The statistical analyses were performed with Stata 12.0 (StataCorp LP, 4905 Lakeway Drive, College Station, TX 77845 USA).

Three-level nested models were specified (individual, class, and school) using the xtmelogit commands to address the dependency between the individuals in the cluster sampling data. Four dependent variables were tested:(1) participating in seven days of MVPA per week (yes/no); (2) at least 60 minutes of MVPA on days in which students reported having MVPA (yes/no); (3) meeting the recommended level of at least 60 minutes of MVPA daily; and (4) meeting the recommendation of no more than 2 hours of leisure-time sedentary behaviours per day (yes/no). Multivariate models were fitted with different levels of adjustment. Model 1 included each type of family support (i.e., verbally encouraging, watching, or involving) separately. Model 2 included three types of family support simultaneously. Model 3 additionally included sex, grade, BMI, and health belief. Model 4 additionally included survey time (2009 or 2011), mother's level of education, and the perceptions of the neighbourhood environment. The tests for linear trend across familial support categories were performed by assigning the midpoint values of each frequency category and treating the variable as continuous in a separate model. We also examined associations between family support and MVPA and sedentary time across gender. Test for interaction were performed by means of likelihood ratio tests to compare multivariate models with and without the interaction terms between family support and gender.

\section{Results}

We excluded students who were unable to engage in PA due to disability, temporary or permanent disease $(\mathrm{n}=895)$ and with missing in any variables $(\mathrm{n}=1090)$. The final analyses included 3811 (52.3\%) boys and 3475 $(47.7 \%)$ girls. As compared with the excluded students, students kept in the analyses were more in Grade 7 and 
with more highly-educated mother. There was no statistically significant difference in sex and BMI. Overall, $31.2 \%$ of students participated in seven days of MVPA per week and $14.6 \%$ had at least 60 minutes of MVPA on days in which students reported having MVPA. Only $9.0 \%$ of students met the recommended level of PA for children - that is, at least 60 minutes of MVPA daily. $63.9 \%$ of students met the recommendation of no more than 2 hours of leisure-time sedentary behaviours per day, with a higher proportion on school days than on weekends. Higher proportions of active students, those who participated in seven days of MVPA per week, had at least 60 minutes of MVPA on days in which they reported having MVPA, met the recommended level of PA and met the recommendation of no more than 2 hours of leisure-time sedentary behaviours per day, received family support almost every day (P value for trend $<0.05$, Table 1 ). The characteristics of the study participants according to familial support were presented in the Table 1.

Multivariate-adjusted analyses showed that neither frequent verbal encouragement nor frequent watching were associated with higher level of MVPA (Figures 1 and 2). Students who reported familial support 1-2 days per week had lower frequency and duration of PA instead as compared with those who reported familial support less than once per week. However, frequent verbal encouragement and watching were associated less leisure-time sedentary behaviours. As compared with students who reported familial support less than once per week, the multivariate-adjusted odds ratios (ORs) for verbal encouragement were 1.13 (95\% CI, 0.95 to 1.36 ) for 1-2 days per week, 1.03 (95\% CI, 0.85 to 1.24 ) for 3-4 days per week, and 1.29 (95\% CI, 1.08 to 1.55) for 5-7 days per week; the respective ORs for watching were 1.20 (95\% CI, 1.05 to 1.38), 1.23 (95\% CI, 1.03 to 1.48 ), and 1.19 (95\% CI, 0.97 to 1.45 ). More details were shown in Additional file 1: Table S1-S4.

Multivariate-adjusted analyses showed that the involvement of family member in the children's activity in most of the days of the week was associated with both greater frequency and longer duration of MVPA and less leisure-time sedentary behaviours. The multivariateadjusted ORs among students who reported familial support 5-7 days per week, as compared with students who reported less than once per week, were 1.25 (95\% CI, 0.95 to 1.66) for at least 60 minutes of MVPA on days in which students reported having MVPA, 1.50 (95\% CI, 1.21 to 1.86) for engaging in seven days of MVPA per week, 1.67 (95\%CI, 1.19 to 2.32$)$ for meeting the recommended level of at least 60 minutes of MVPA daily, and $1.48(95 \% \mathrm{CI}, 1.19$ to 1.84$)$ for no more than 2 hours of leisure-time sedentary behaviours per day (Figures 1 and 2). More details were shown in Additional file 1: Table S1-S4.

\section{Discussions}

In this large sample size study of Chinese urban adolescents, we found that less than $10.0 \%$ of students met the recommended level of PA for children - that is, at least 60 minutes of MVPA daily. This study is also one of the few studies to explore the relationship between the family support and children's PA and sedentary behaviours in Chinese urban adolescents. We found that family members involving themselves in the children's activity had the most significant influence on children's behaviours as compared with other types of supports such as verbally encouraging and observing children's activity. Involvement of family members in the children's activity almost every day was associated with higher level of MVPA and less leisure-time sedentary behaviours.

It has been suggested that strong family support increases children's self-efficacy to overcome barriers to being physically active [8]. Self-efficacy refers to a person's belief in his or her ability to succeed in a particular situation [20]. Children who have strong selfefficacy believe that they can successfully overcome obstacles to physical fitness and skills. Persuasive information, as in verbal encouragement from parents, can raise self-efficacy, but its effects can be transitory if subsequent performance turns out differently [21]. In contrast with verbally expressed expectation, the effects of parental expectations on children's behaviours seem greatest when a high level of parent involvement exists [21]. The familial involvement in PA may boost children's efficacy to improve MVPA, help children develop skills necessary to be physically active, and provide logistical support $[8,22]$.

Sedentary behaviours have emerged as an important health risk factor independent of MVPA [23,24]. Although verbally encouraging and watching were not associated with a higher level of MVPA in this study, they were observed to be associated with less leisure-time sedentary behaviours. One possible explanation is that some bouts of light activity have replaced sedentary time under the familiar pressure on children. Several previous studies have reported that sedentary time was inversely correlated with light-intensity $\mathrm{PA}$ such as walking and playing $[25,26]$. While increasing MVPA should still be a public health priority, breaks in sedentary time and light-intensity PA may also provide a beneficial adjunct to the current $60 \mathrm{~min} /$ day of MVPA recommendation $[27,28]$.

In the current study, we hypothesised that familial support such as verbal encouragement, watching, and involvement is one of the most important motivations for children to get outdoors in the leisure time at home. The more frequent the family members are concerned about their children's physical activity in whatever way, the more physically active children are. We used the 
Table 1 Characteristics by family influence group $(\mathrm{N}=7286)^{*}$

\begin{tabular}{|c|c|c|c|c|c|c|c|c|c|c|c|c|c|c|}
\hline \multicolumn{5}{|c|}{ Verbal encouragement (days/week) } & \multicolumn{5}{|c|}{ Watching (days/week) } & \multicolumn{5}{|c|}{ Involvement (days/week) } \\
\hline Less than weekly & $1-2$ & 3-4 & $5-7$ & $P$ & Less than weekly & $1-2$ & 3-4 & $5-7$ & $P$ & Less than weekly & $1-2$ & 3-4 & $5-7$ & $\mathbf{P}$ \\
\hline
\end{tabular}

\section{Grade, n (\%)}

Grade 7

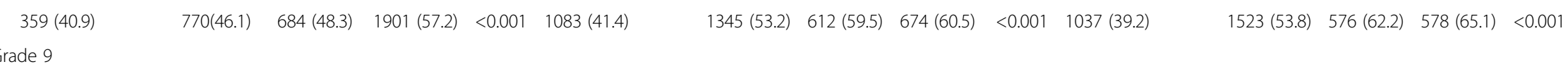

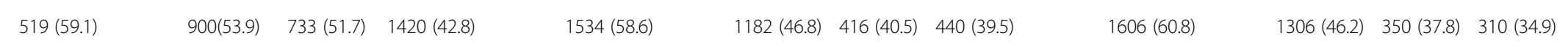

\section{Mother's level of education, $\mathrm{n}(\%)$}

Low

\begin{tabular}{|c|c|c|c|c|c|c|c|c|c|c|c|c|}
\hline ) & 552 (33.1) & $421(297)$ & $848(25.5)$ & $<0.001$ & $903(345)$ & $709(281)$ & $248(24.1)$ & 200 (1951) & \multicolumn{2}{|c|}{$<0.001 \quad 912(345)$} & $752(266)$ & $247(267) \quad 229(258)$ \\
\hline
\end{tabular}

Middle

\begin{tabular}{|c|c|c|c|c|c|c|c|c|c|c|c|}
\hline 218 (24.8) & $403(24.1)$ & $358(25.3)$ & $763(23.0)$ & $644(24.6)$ & $616(24.4)$ & $232(22.6)$ & $250(22.4)$ & $667(25.2)$ & $663(23.4)$ & $210(22.7)$ & $202(22.7)$ \\
\hline & & & & & & & & & & & \\
\hline 295 (33.6) & 641 (38.4) & 589 (41.6) & 1575 (47.4) & $948(36.2)$ & 1103 (43.6) & $506(49.2)$ & $543(48.7)$ & 946 (35.8) & $1302(46.0)$ & 431 (46.5) & $421(47.4)$ \\
\hline
\end{tabular}

Not sure

$46(5.2) \quad 74(4.4) \quad 49(3.5) \quad 135(4.1) \quad 122(4.7)$

BMI $\left(\mathbf{k g} / \mathbf{m}^{2}\right), \bar{X} \pm S d$

\section{$20.0 \pm 3.0$}

\section{7 days/wk}

$$
\text { 283(32.2) } \quad 364(21.8) \quad 365(25.8) \quad 1260(37.9) \quad<0.001 \quad 776(29.7)
$$

At least 60 minutes/day

$$
168(19.1) \quad 172(10.3) \quad 169(11.9) \quad 557(16.8) \quad<0.001 \quad 419(16.0)
$$

At least 60 minutes/day, 7 days/wk

$$
\text { 99(11.3) } \quad \text { 86(5.1) } \quad 92(6.5) \quad 376(11.3) \quad<0.001 \quad 246(9.4)
$$

\section{Sedentary behaviours (no more than 2 hours), $\mathrm{n}(\%)$}

On school days

$$
\begin{array}{llllll}
721(82.1) & 1445(86.5) & 1250(88.2) & 3019(90.9) & <0.001 & 2266(86.6)
\end{array}
$$

On weekends

296(33.7)

$614(36.8) \quad 497(35.1) \quad 1409(42.4) \quad<0.001 \quad 905(34.6)$

During the week

638(72.7)

1354(81.1) $\quad 1170(82.6) \quad 2881(86.8) \quad<0.001 \quad 2073(79.2)$

$\begin{array}{lllllllllll}99(3.9) & 42(4.1) & 41(3.7) & & 118(4.5) & 112(4.0) & 38(4.1) & 36(4.1) & \\ 20.0 \pm 3.3 & 20.1 \pm 3.5 & 20.0 \pm 3.5 & 0.003 & 20.3 \pm 3.3 & 20.0 \pm 3.4 & 20.0 \pm 3.5 & 19.9 \pm 3.4 & <0.001 \\ & & & & & & & & \\ 642(25.4) & 313(30.4) & 541(48.6) & <0.001 & 770(29.1) & 782(27.6) & 280(30.2) & 440(49.5) & <0.001 \\ 278(11.0) & 131(12.7) & 238(21.4) & 0.007 & 422(16.0) & 341(12.1) & 104(11.2) & 199(22.4) & 0.001 \\ 147(5.8) & 75(7.3) & 185(16.6) & <0.001 & 247(9.3) & 182(6.4) & 60(6.5) & 164(18.5) & <0.001 \\ & & & & & & & & \\ 2252(89.1) & 915(89.0) & 1002(89.9) & 0.004 & 2286(86.5) & 2537(89.7) & 813(87.8) & 799(90.0) & 0.015 \\ & & & & & & & & & \\ 989(39.1) & 395(38.4) & 527(47.3) & <0.001 & 906(34.3) & 1091(38.6) & 385(41.6) & 434(48.9) & <0.001 \\ 2125(84.1) & 880(85.6) & 965(86.6) & <0.001 & 2081(78.7) & 2408(85.1) & 784(84.7) & 770(86.7) & <0.001\end{array}$


Table 1 Characteristics by family influence group $(\mathbf{N}=\mathbf{7 2 8 6}) *$ (Continued)

\section{Health belief (at least 60 minutes/day), $\mathrm{n}(\%)$}
293(33.4)

533(31.9) $\quad 435(30.7) \quad 1313(39.5) \quad<0.001 \quad 903(34.5)$

808(32.0)

397(38.6) $\quad 466(41.8)$

$<0.001944(35.7)$

$957(33.8) \quad 322(34.8) \quad 351(39.5) \quad 0.07$

Opinion of neighbourhood environment, $n$ (\%)

Busy traffic
114(13)
$188(11.3) \quad 140(9.9) \quad 286(8.6)$
$<0.001 \quad 304(11.6)$
256(10.1)
$81(7.9) \quad 87(7.8)$
$<0.001 \quad 296(11.2)$
$287(10.1) \quad 74(8.0) \quad 71(8.0)$
$<0.001$

Easily available parks or gyms

499(56.8)

$\begin{array}{lllll}998(59.8) & 927(65.4) & 2385(71.8) & <0.001 & 1564(59.8)\end{array}$

$1678(66.4) \quad 726(70.6) \quad 841(75.5) \quad<0.001 \quad 1602(60.6)$

$1870(66.1) \quad 654(70.6) \quad 683(76.9) \quad<0.001$

Safe during the day

$$
\text { 594(67.7) }
$$

$1896(75) \quad 794(77.2) \quad 873(78.4) \quad<0.001 \quad 1875(70.9)$

$2133(75.4) \quad 72478.2) \quad 691(77.8) \quad<0.001$

*Watching: Families watched the child participate in PA. Verbal encouragement: Families encouraged the child to do PA. Involvement: Families involved the child in PA. MVPA: Moderate to vigorous intensity physical activity; BMI: Body Mass Index; Sd: Standard deviation; $\bar{X}$ : Mean. Family influence was recoded as the continuous variable: (1) less than weekly: 0; (2) 1-2 days/week: 1.5; (3) 3-4 days/week: 3.5; (4) 5-7 days/week: 6. P: P-values for trend represent family influence group comparison. 


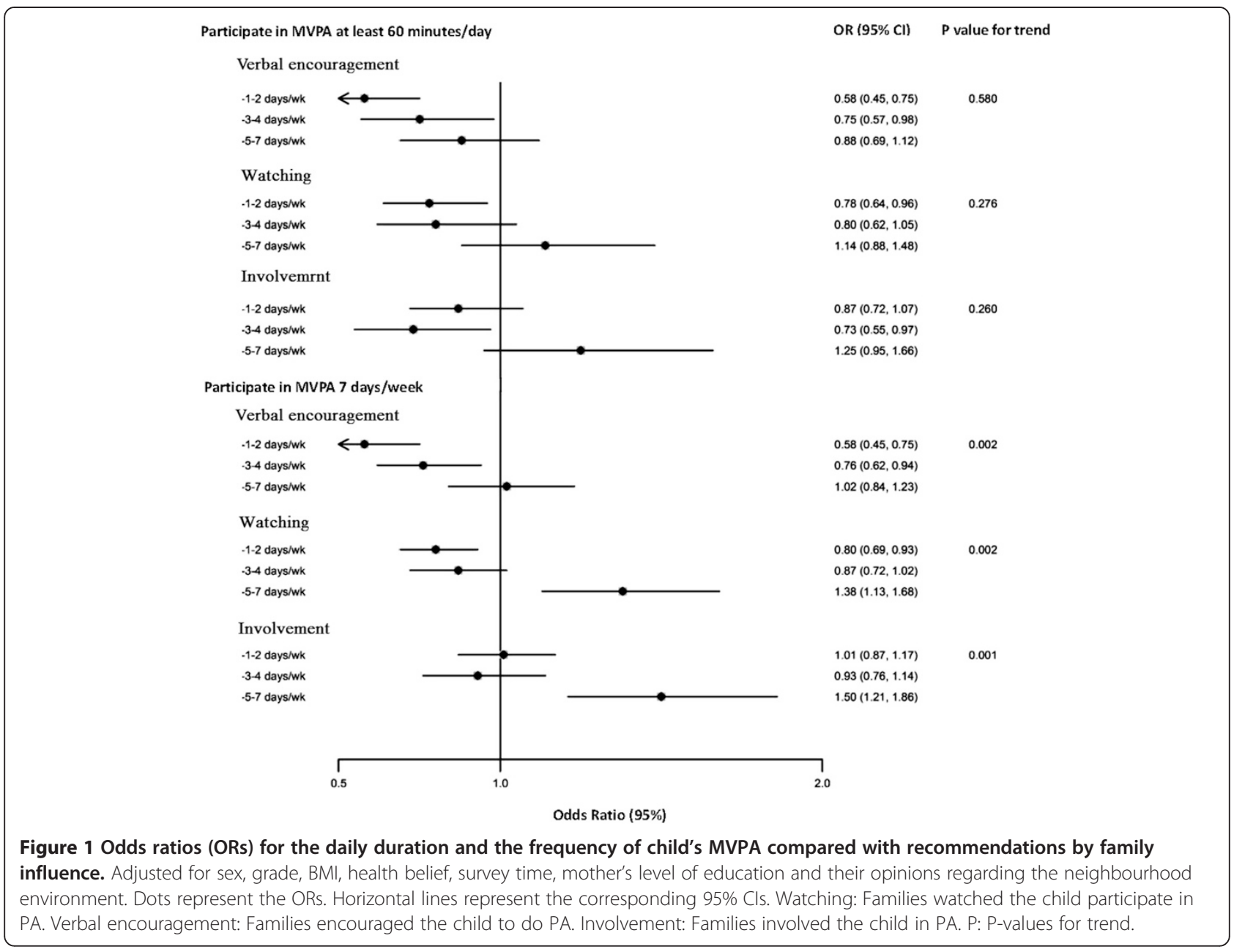

weekly frequency as a measurement for the strength of familial support. Familial support could also manifest itself in other ways. For example, parents watch the exercise sessions which they book for their children or involve themselves in planned school events. In this situation, the proportion of parents attending scheduled events would be a better measurement for the strength of familial support.

This study acknowledges a few limitations that should be noted. First, given the cross-sectional nature of this study, it is not possible for us to determine the temporal relationship between familial support and children's behaviours. Two-way causal relationship might exist. Stronger family support is associated with more physically active children, while for physically active children who like and specialize in sports, parents may save the efforts to push their children into playing sports. In the current study, we could not exclude the possibility that some physically active children consequently received less familial support which might have attenuated the positive influence of familiar support on children's behaviours toward null. Second, participants were more likely from educated households, possibly resulting in a potential bias [13]. However, the lack of family involvement in children's PA who were from educated household highlights the importance of increasing family involvement to promote children's MVPA and to reduce sedentary time. Third, residual confounding by other unmeasured or unknown factors such as children's preference for PA and parental level of PA remains possible although we have carefully adjusted for some potential factors for children's PA. Forth, the levels of MVPA, sedentary time, and family support were self-reported; therefore, some measurement error is inevitable. However, relevant survey instruments have been shown to have good reliability and validity in diverse populations [17,29-31].

\section{Conclusion}

In summary, in this large sample size study of Chinese urban adolescents, we found that family members involving themselves in the children's activity had the 


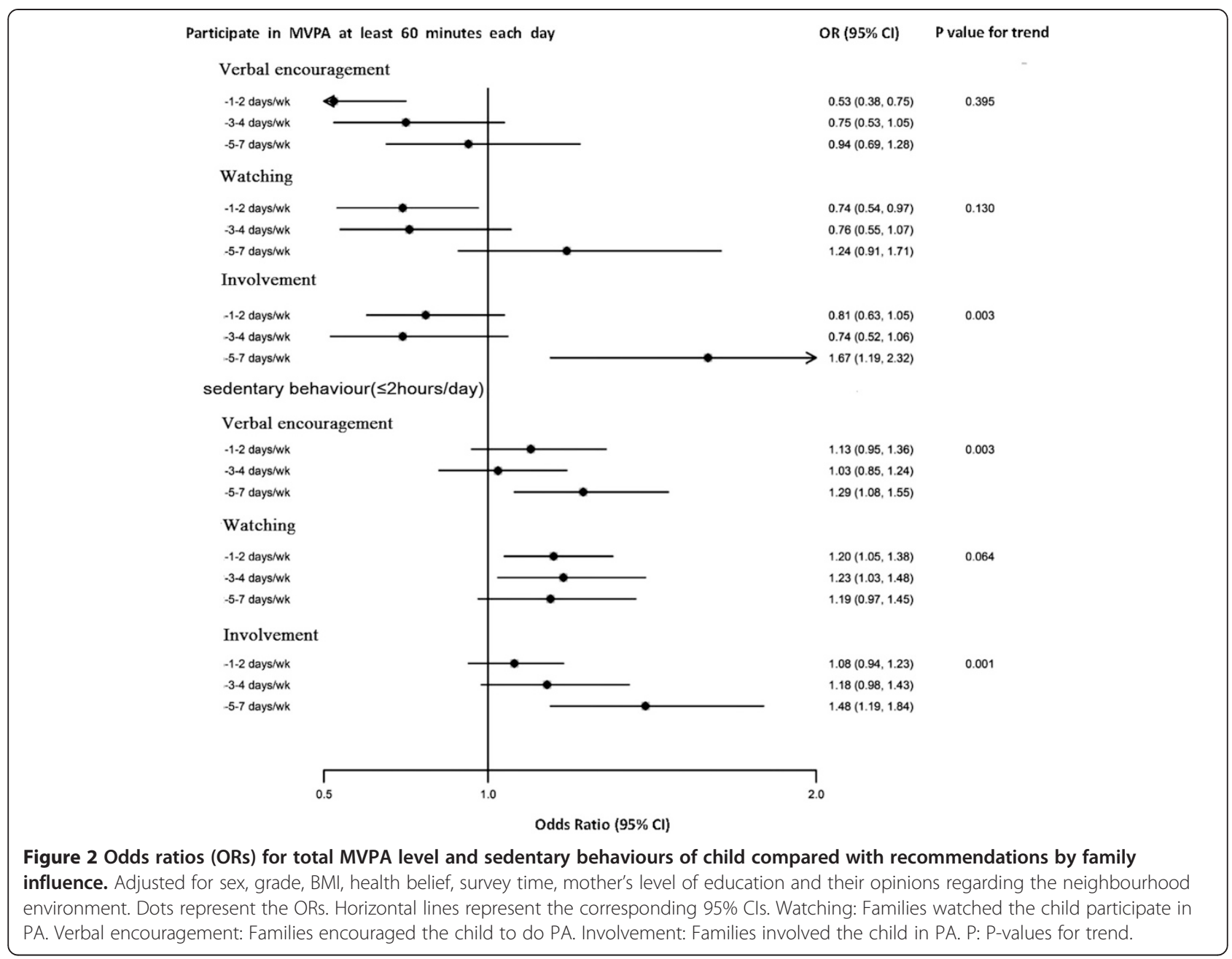

most significant influence on children's behaviours as compared with other types of supports such as verbally encouraging and observing children's activity. Any type of familial support including verbally encouraging, watching, and involving had effects on reducing leisure-time sedentary behaviours.

\section{Additional file}

Additional file 1: Table S1. The association between family influence and the duration of MVPA per day $(n=7286)$. OR $(95 \% \mathrm{Cl})$ for the duration of MVPA of child (at least 60 minutes/day or not) by family influence. Multivariate models were adjusted for the following factors: (1) model 1 included certain family influence (encourage, watch or involve in); (2) model 2 included all family influence (encourage, watch and involve in); (3) model 3 additionally included sex, grade, BMI and health belief of daily PA time; (4) model 4 additionally included survey time, mother's level of education and opinion regarding neighbourhood environment. Based on model 4, three-level nested models were specified (individual, class and school) with equal random effects variance and covariance being zero, and the coefficient is the SD $(95 \% \mathrm{CI})$ of random intercept. Family influence was recoded as the continuous variable: (1) less than weekly: 0; (2) 1-2 days/week: 1.5; (3) 3-4 days/week: 3.5; (4) 5-7 days/week: 6. P-values for trend represent family influence group comparison. Table S2. The association between family influence and the frequency of MVPA ( $n=7286)$. OR $(95 \%$ Cl) for the frequency of MVPA of child (7 days/week or not) by family influence. Table S3. The association between family influence and total MVPA per week $(n=7286)$. OR (95\% Cl) for the total level of MVPA of child (at least 60 minutes each day or not) by family influence. Table S4. The association between family influence and sedentary time $(n=7286)$. OR $(95 \% \mathrm{Cl})$ for sedentary time of child (at least 60 minutes/day or not) by family influence.

\section{Abbreviations}

MVPA: Moderate to vigorous intensity physical activity; PA: Physical activity; BMl: Body mass index; Sd: Standard deviation; $\bar{X}$ : Mean; OR: Odds ratio; $\mathrm{Cl}$ : Confidence interval; Xtmelogit: Multilevel mixed-effects logistic regression.

\section{Competing interests}

The authors declare that they have no competing interests.

\section{Authors' contributions}

$X W$ drafted the manuscript. $J$ developed the sampling design and reviewed the manuscript. XW and LJ undertook the statistical analysis. QML and YJR organized the field work. LML developed whole research designs, contributed to the interpretation of the results and reviewed the manuscript. All authors read and approved the final manuscript. 


\section{Authors' information}

XW is a master, at the Department of Epidemiology and Biostatistics, School of Public Health, Peking University Health Science Centre (PUHSC) in Beijing, China. $J$ is an associate professor, at the Department of Epidemiology and Biostatistics, School of Public Health, Peking University Health Science Centre (PUHSC) in Beijing, China. QML is a director at the Division for Chronic and Non-Communicable Disease Control and Prevention, Hangzhou Centre for Disease Control and Prevention in Hangzhou, China. YJR is a program coordinator at the Division for Chronic and Non-Communicable Disease Control and Prevention, Hangzhou Centre for Disease Control and Prevention in Hangzhou, China. LML is a professor at the Department of Epidemiology and Biostatistics, School of Public Health, PUHSC and is also one of the Hangzhou Qianjiang Scholars.

\section{Acknowledgements}

Community Interventions for Health $(\mathrm{CH})$ is supported by a registered UK charity, the Oxford Health Alliance $(\mathrm{OxHA})$ and facilitated by MATRIX Public Health Solutions Inc. For a full list of donors supporting OxHA please go to http://www.oxha.org. We are deeply indebted the study participants for their participation and to all the staff in districts of Xiacheng, Gongshu, Xihu in Hangzhou for their efforts that made the study possible.

\section{Author details}

${ }^{1}$ Department of Epidemiology and Biostatistics, School of Public Health, Peking University Health Science Centre, Beijing 100191, China. ${ }^{2}$ Division for Chronic and Non-Communicable Disease Control and Prevention, Hangzhou Centre for Disease Control and Prevention, Mingshi Road, Jianqiao Town, Hangzhou 310021, China. ${ }^{3}$ Division for Chronic and Non-Communicable Disease Control and Prevention, Hangzhou Centre for Disease Control and Prevention, Mingshi Road, Jianqiao Town, Hangzhou 310021, China. ${ }^{4}$ Department of Epidemiology and Biostatistics, School of Public Health, Peking University Health Science Centre, Beijing 100191, China. ${ }^{5}$ Department of Epidemiology and Biostatistics, School of Public Health, Peking University Health Science Centre, Beijing 100191, China.

Received: 5 November 2014 Accepted: 27 February 2015

Published online: 25 March 2015

\section{References}

1. Saunders TJ, Chaput JP, Tremblay MS. Sedentary behaviour as an emerging risk factor for cardiometabolic diseases in children and youth. Can J Diab. 2014;38:53-61.

2. Lien AS, Cho YH, Tsai JL. Effectiveness evaluation of healthy lifestyle interventions in childhood obesity prevention: a systematic review]. Hu Li Za Zhi. 2013;60:33-42.

3. Teychenne M, York R. Physical activity, sedentary behavior, and postnatal depressive symptoms: a review. Am J Prev Med. 2013;45:217-27.

4. Bi Y, Wang T, Xu M, Xu Y, Li M, Lu J, et al. Advanced research on risk factors of type 2 diabetes. Diabetes Metab Res Rev. 2012;28 Suppl 2:32-9.

5. World Health Organization: Global Strategy on Diet, Physical Activity and Health. 2015. http://www.who.int/dietphysicalactivity/ factsheet_young_people/en/. Accessed 19 Jan 2015.

6. Ma GS, Luan DC, Hu XQ, Cui CH, Liu AL, Li YP, et al. Present Status of Physical Activity of Chinese[J]. Danone Inst China. 2005;03:24-7.

7. Li BH, Wu SS, Wang HJ, Ma J, Zhang SW. The investigation on sports of child and adolescent in five cities in China. In: Proceedings of the 11th Annual Symoposium Of Danone Institute China. 2008. p. 27-8.

8. Trost SG, Sallis JF, Pate RR, Freedson PS, Taylor WC, Dowda M. Evaluating a model of parental influence on youth physical activity. Am J Prev Med. 2003;25:277-82.

9. Vander Ploeg KA, Kuhle S, Maximova K, McGavock J, Wu B, Veugelers PJ. The importance of parental beliefs and support for pedometer-measured physical activity on school days and weekends days among Canadian children. BMC Public Health. 2013;13:1132.

10. Heitzler CD, Martin SL, Duke J, Huhman M. Correlates of physical activity in a national sample of children aged 9-13 years. Prev Med. 2006;42:254-60.

11. Springer AE, Kelder SH, Hoelscher DM. Social support, physical activity and sedentary behavior among 6th-grade girls: a cross-sectional study. Int J Behav Nutr Physical Act. 2006;3:8.
12. Adkins S, Sherwood NE, Story M, Davis M. Physical activity among African-American girls: the role of parents and the home environment. Obesity Res. 2004;12(Suppl):38s-45.

13. Eime RM, Harvey JT, Craike MJ, Symons CM, Payne WR. Family influence and ease of access link socio-economic status and sports club membership in adolescent girls: a mediation study. Int J Behav Nutr Physical Act. 2013;10:50.

14. Prochaska JJ, Rodgers MW, Sallis JF. Association of parent and peer support with adolescent physical activity. Res Q Exerc Sport. 2002;73:206-10.

15. Duffany KOC, Finegood DT, Matthews D, McKee M, Venkat Narayan KM, Puska $\mathrm{P}$, et al. Community Interventions for Health $(\mathrm{CIH})$ : A novel approach to tackling the worldwide epidemic of chronic diseases. CVD Prev Control. 2011;6:47-56

16. Health Behaviour in School-age Children. 2002. http://www.hbsc.org/. Accessed 19 Jan 2015

17. Liu Y, Wang M, Tynjala J, Lv Y, Villberg J, Zhang Z, et al. Test-retest reliability of selected items of Health Behaviour in School-aged Children (HBSC) survey questionnaire in Beijing, China. BMC Med Res Methodol. 2010;10:73.

18. World Health Organization: Global Strategy on Diet, Physical Activity and Health. 2015. http://www.who.int/dietphysicalactivity/pa/en/index.html. Accessed 19 Jan 2015.

19. Participation: Sedentary Behaviour Guidelines for youth 12-17 years. 2013. http://www.participaction.com/get-informed/physical-activity-guidelines/ sedentary-behaviour-guidelines/sedentary-behaviour-guidelines-12-17/. Accessed 19 Jan 2015.

20. Kendra C: What Is Self-Efficacy? 2015. http://psychology.about.com/od/ theoriesofpersonality/a/self_efficacy.htm. Accessed 19 Jan 2015.

21. Dale HS, Judith LM. Self-efficacy development in adolescences. In: Frank P, Timothy CU, editors. Self-Efficacy Beliefs of Adolescents. Charlotte, North Carolina: Information Age Publishing; 2005.

22. Bandura A. Self-efficacy: toward a unifying theory of behavioral change. Psychol Rev. 1977;84:191-215.

23. Sallis JF, Alcaraz JE, McKenzie TL, Hovell MF. Predictors of change in children's physical activity over 20 months. Variations by gender and level of adiposity. Am J Prev Med. 1999;16:222-9.

24. Biddle SJ, Asare M. Physical activity and mental health in children and adolescents: a review of reviews. Br J Sports Med. 2011;45:886-95.

25. Lowry R, Wechsler H, Galuska DA, Fulton JE, Kann L. Television viewing and its associations with overweight, sedentary lifestyle, and insufficient consumption of fruits and vegetables among US high school students: differences by race, ethnicity, and gender. J Sch Health. 2002;72:413-21.

26. Strauss RS, Rodzilsky D, Burack G, Colin M. Psychosocial correlates of physical activity in healthy children. Arch Pediatr Adolesc Med. 2001;155:897-902.

27. Healy GN, Dunstan DW, Salmon J, Cerin E, Shaw JE, Zimmet PZ, et al. Breaks in sedentary time: beneficial associations with metabolic risk. Diabetes Care. 2008;31:661-6

28. Carson V, Ridgers ND, Howard BJ, Winkler EA, Healy GN, Owen N, et al. Light-intensity physical activity and cardiometabolic biomarkers in US adolescents. PLoS One. 2013;8:e71417.

29. Ridgers ND, Timperio A, Crawford D, Salmon J. Validity of a brief self-report instrument for assessing compliance with physical activity guidelines amongst adolescents. J Sci Med Sport. 2012;15:136-41.

30. Rey-Lopez JP, Vicente-Rodriguez G, Ortega FB, Ruiz JR, Martinez-Gomez D, De Henauw S, et al. Sedentary patterns and media availability in European adolescents: The HELENA study. Prev Med. 2010;51(1):50-5.

31. Sallis JF, Taylor WC, Dowda M, Freedson PS, Pate RR. Correlates of vigorous physical activity for children in grades 1 through 12: Comparing parent- reported and objectively measured physical activity. Pediatr Exerc Sci. 2002;14:30-44. 